\title{
Direct and mixing-induced CP violation in charmless two-body B decays.
}

\section{Denis Derkach*}

INFN-Bologna/CERN

E-mail: denis.derkachecern.ch

\begin{abstract}
The recent analyses performed by the LHCb collaboration in the sector of the charmless two-body $B$-decays. The following analyses are included: time-integrated $C P$ asymmetry measurement of $B_{d} \rightarrow K \pi$ and $B_{s} \rightarrow \pi K$ decays, time-dependent measurements of $B_{d} \rightarrow \pi \pi$ and $B_{s} \rightarrow K K$ decays, effective lifetime measurements of $B_{s} \rightarrow K K$ decays, and triple asymmetries of $B_{S} \rightarrow \phi \phi$.
\end{abstract}

The XIth International Conference on Heavy Quarks and Leptons 11-15 June, 2012

Prague, The Czech Republic

\footnotetext{
* Speaker.
} 


\section{Introduction}

The family of the $B$ hadron decays into pairs of charmless mesons or baryons includes a very rich set of channels. These channels can give an important insight into the Standard Model (SM) dynamics and a possibility to discover effects beyond the SM. These effects, commonly known as New Physics (NP), may show up as virtual contributions of new particles inside the loops of the strong and electroweak penguin graphs contributing to the amplitudes of such decays. These proceedings describe the measurements that provide the information on the magnitude of NP effects performed with the LHCb detector at the Large Hadron Collider, CERN.

$\mathrm{LHCb}[1]$ is a dedicated detector for $b$ and $c$-physics precision measurements at the LHC that is designed as a single arm forward spectrometer covering the pseudorapidity range $2<\eta<5$, which ensures a high geometric efficiency for detecting the decay particles from signal $b$ hadron together with the tracks from the rest of the event that can be used to tag the flavor. Such detector design offers further advantages: $b$ hadrons are expected to have a hard momentum spectrum in the forward region; their average momentum is $80 \mathrm{GeV} / c$, corresponding to approximately $7 \mathrm{~mm}$ mean decay distance, which facilitates the separation between primary and decay vertices. This property, coupled to the excellent vertex resolution capabilities, allows proper time to be measured with a few percent uncertainty, which is crucial for studying $C P$ violation and oscillations of $B_{s}$ mesons. The data is taken with two polarities of magnet (approximately, 50\% of the time each). This allows to cancel some systematic effects.

In the following I report the results of several analyses studying the charmless two-body decays of $b$ mesons. The analyses are based on the data collected by LHCb in 2011: the time-integrated study of $B_{d} \rightarrow K \pi$ and $B_{s} \rightarrow \pi K$ is performed on $0.35 \mathrm{fb}^{-1}$ of data, the time-dependent $B_{d} \rightarrow \pi \pi$ and $B_{s} \rightarrow K K$ use $0.69 \mathrm{fb}^{-1}$, all the others use the full 2011 data sample $\left(1 \mathrm{fb}^{-1}\right)$. The main common part of all the various analyses is the low-level event selection, which is performed by the two-level hadronic trigger of LHCb. The first level (Level 0) is based on custom electronic boards, selecting events with high transverse energy clusters in the hadronic calorimeter. The second level, so called High Level Trigger (HLT), is software-based and selects events with at least one track with high transverse momentum and large impact parameter with respect to all reconstructed primary vertices. Another common feature of analyses is the usage of LHCb particle identification (PID) exploiting the capabilities of the two RICH detectors in order to separate kaons, pions and protons.

\section{Time-integrated study of $B_{d} \rightarrow K \pi$ and $B_{s} \rightarrow \pi K$}

This analysis [2] uses the events that are extracted from the triggered data using two different off-line selections, each one targeted to achieve the best sensitivity on

$$
A_{C P}=[\Gamma(\bar{B} \rightarrow \bar{f})-\Gamma(B \rightarrow f)] /[\Gamma(\bar{B} \rightarrow \bar{f})+\Gamma(B \rightarrow f)]
$$

for $B_{d} \rightarrow K \pi$ and $B_{s} \rightarrow \pi K$ events.

The strategy used to optimize the cuts is divided into two steps. In the first step we define the kinematic cuts against the combinatorial background, selecting in an inclusive way the $B$-meson decaying into two body candidates, without using any PID information and assigning by default the pion-mass hypothesis to all charged tracks. The two kinematic selections use the same set of cuts, 
Table 1: Summary of selection criteria adopted for the measurement of $A_{C P}\left(B_{d} \rightarrow K \pi\right)$ and $A_{C P}\left(B_{s} \rightarrow K \pi\right)$.

\begin{tabular}{lcc}
\hline \hline Variable & $A_{C P}\left(B_{d} \rightarrow K \pi\right)$ & $A_{C P}\left(B_{s} \rightarrow K \pi\right)$ \\
\hline Track quality $\chi^{2} /$ ndf & $<3$ & $<3$ \\
Track $p_{\mathrm{T}}[\mathrm{GeV} / c]$ & $>1.1$ & $>1.2$ \\
$\operatorname{Track} d_{\mathrm{IP}}[\mathrm{mm}]$ & $>0.15$ & $>0.20$ \\
$\max \left(p_{\mathrm{T}}^{K}, p_{\mathrm{T}}^{\pi}\right)[\mathrm{GeV} / c]$ & $>2.8$ & $>3.0$ \\
$\max \left(d_{\mathrm{IP}}^{K}, d_{\mathrm{IP}}^{\pi}\right)[\mathrm{mm}]$ & $>0.3$ & $>0.4$ \\
$d_{\mathrm{CA}}[\mathrm{mm}]$ & $<0.08$ & $<0.08$ \\
$p_{\mathrm{T}}^{B}[\mathrm{GeV} / c]$ & $>2.2$ & $>2.4$ \\
$d_{\mathrm{IP}}^{B}[\mathrm{~mm}]$ & $<0.06$ & $<0.06$ \\
$t_{\pi \pi}[\mathrm{ps}]$ & $>0.9$ & $>1.5$ \\
\hline \hline
\end{tabular}

but with different thresholds. They select pairs of oppositely charged tracks with high transverse momentum and large impact parameter with respect to all reconstructed primary vertices, fitted to a common vertex displaced from the related primary vertex. The following discriminating variables are used: the quality of the online-reconstructed tracks $\left(\chi^{2}\right.$ per degree of freedom), their transverse momenta $\left(p_{\mathrm{T}}\right)$ and their impact parameters $\left(d_{\mathrm{IP}}\right.$, defined as the distance between the reconstructed trajectory of the track and the $p p$ collision vertex); the distance of closest approach of the decay products of the $B$ meson candidate $\left(d_{\mathrm{CA}}\right)$, its transverse momentum $\left(p_{\mathrm{T}}^{B}\right)$, its impact parameter $\left(d_{\mathrm{IP}}^{B}\right)$ and the decay time in its rest frame $\left(t_{\pi \pi}\right.$, calculated assuming the decay into $\left.\pi^{+} \pi^{-}\right)$. Only $B$ candidates within the $\pi \pi$ invariant mass range $4.7-5.9 \mathrm{GeV} / c^{2}$ are accepted. A summary of the two distinct sets of selection criteria is reported in Table 1.

In the second step, two sets of PID cuts are defined (one for each set of optimized kinematic cuts). The guiding principle to identify the PID selection criteria is to limit the total amount of cross-feed backgrounds under the $B_{d} \rightarrow K^{+} \pi^{-}$and $B_{s} \rightarrow \pi^{+} K^{-}$mass peaks to the same level as the corresponding combinatorial background. The calibration of the PID observables is a crucial aspect of this analysis, as it is the only variable allowing us to discriminate between the various decay modes. Hence, in order to determine the amount of cross-feed backgrounds for a given channel, the relative efficiencies of the PID selection cuts, employed to identify the specific final state of interest, play a key role. For the calibration of the PID performance with use a big sample of $D^{*}$-tagged $D^{0} \rightarrow K \pi$ events. Since the RICH PID information is momentum dependent, the distributions obtained from calibration samples are reweighted according to the momentum distributions of $B$ daughter tracks observed in data.

We perform unbinned maximum likelihood fits to the mass spectra of events passing the optimized offline selections for the measurements of $A_{C P}\left(B_{d} \rightarrow K \pi\right)$ or $A_{C P}\left(B_{s} \rightarrow K^{-} \pi^{+}\right)$. The $B_{d} \rightarrow K \pi$ and $B_{s} \rightarrow K \pi$ signal components are described by single Gaussian functions convolved with a function which describes the effect of final state radiation on the mass lineshape [3]. The background due to partially reconstructed three-body $B$ decays is parametrized by means of an ARGUS function [4] convolved with a Gaussian resolution function. The combinatorial background is modeled by an exponential and the shapes of the cross-feed backgrounds, mainly due to $B_{d} \rightarrow \pi^{+} \pi^{-}$and $B_{s} \rightarrow K^{+} K^{-}$decays with one misidentified particle in the final state, are obtained 

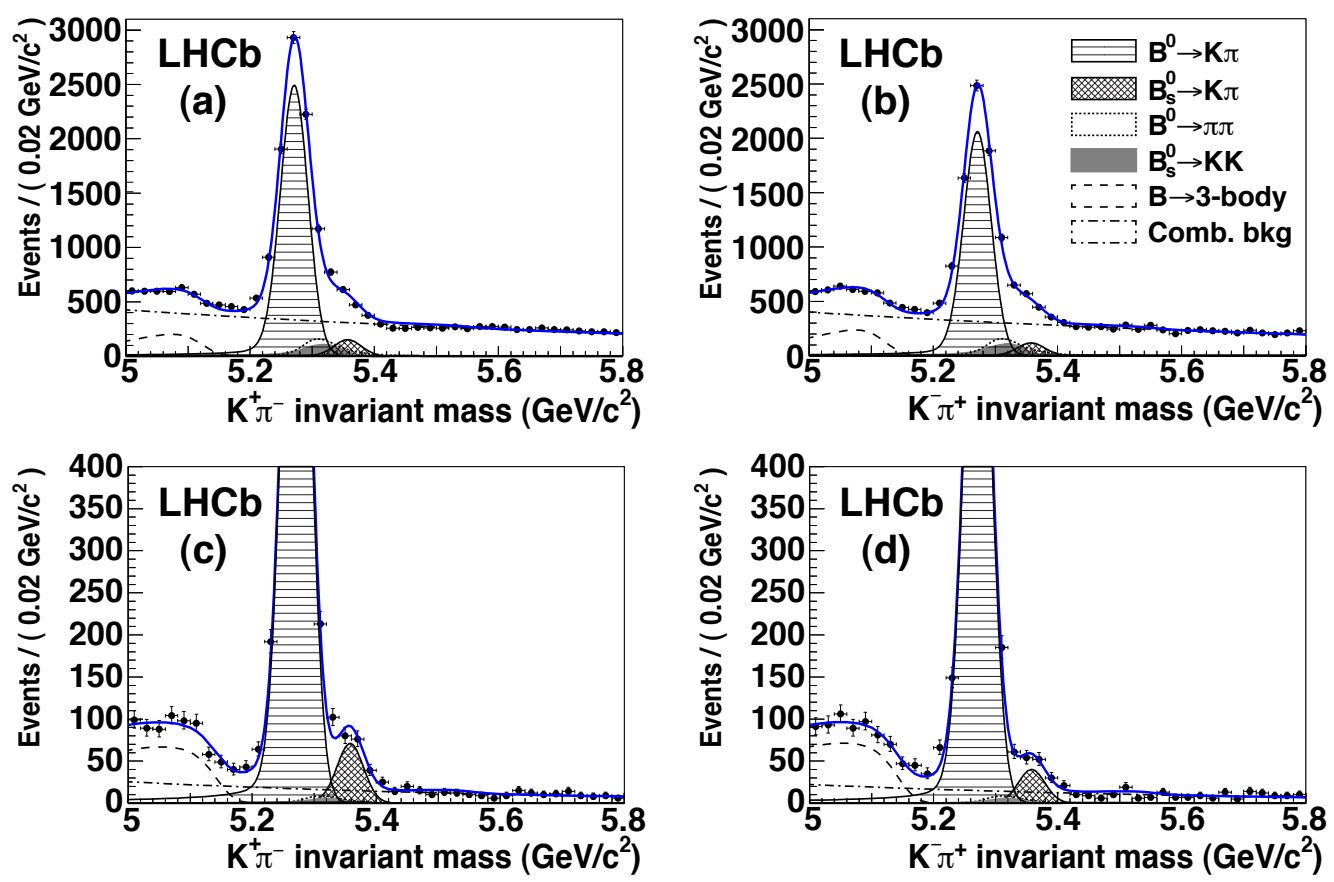

Figure 1: Invariant $K \pi$ mass spectra obtained using the event selection adopted for the best sensitivity on (a, b) $A_{C P}\left(B_{d} \rightarrow K \pi\right)$ and (c, d) $A_{C P}\left(B_{s} \rightarrow K \pi\right)$. Plots (a) and (c) represent the $K^{+} \pi^{-}$invariant mass whereas plots (b) and (d) represent the $K^{-} \pi^{+}$invariant mass. The results of the unbinned maximum likelihood fits are overlaid. The main components contributing to the fit model are also shown.

from Monte Carlo simulations. The $B_{d} \rightarrow \pi^{+} \pi^{-}$and $B_{s} \rightarrow K^{+} K^{-}$cross-feed background yields are determined from fits to the $\pi^{+} \pi^{-}$and $K^{+} K^{-}$mass spectra respectively, using events selected by the same offline selection as the signal and taking into account the appropriate PID efficiency factors. The $K^{+} \pi^{-}$and $K^{-} \pi^{+}$mass spectra for the events passing the two offline selections are shown in Fig. 1.

From the two mass fits we determine the signal yields the raw yield asymmetries $A_{\text {raw }}\left(B_{d} \rightarrow\right.$ $K \pi)=-0.095 \pm 0.011$ and $A_{\text {raw }}\left(B_{s} \rightarrow K \pi\right)=0.28 \pm 0.08$, where the uncertainties are statistical only. We also measure the yields to be $N\left(B_{d} \rightarrow K \pi\right)=13250 \pm 150$ and $N\left(B_{s} \rightarrow K \pi\right)=314 \pm 27$. In order to determine the $C P$ asymmetries from the observed raw asymmetries we first take into account effects induced by the detector acceptance and event reconstruction, as well as due to strong interactions of final state particles with the detector material. This is measured with the help $D^{*} \rightarrow D^{0}(\rightarrow K \pi) \pi$ decays reweighted to have the same momentum distribution. Another correction to be introduced is the production asymmetry of $B$ mesons. This is studied using $B_{d} \rightarrow$ $J / \psi K^{* 0}$ decays taking into account the oscillation frequencies of $B_{d}$ and $B_{s}$. Since the $B_{s}$ meson oscillates quickly, the effect of production asymmetry $A_{C P}$ is strongly suppressed.

The systematic uncertainties of the measurement take into account the PID calibration, final state radiation, possible imperfections of fit model and error of the instrumental and production asymmetry measuremnts.

In conclusion we obtain the following measurements of the $C P$ asymmetries:

$$
A_{C P}\left(B_{d} \rightarrow K \pi\right)=-0.088 \pm 0.011 \text { (stat) } \pm 0.008 \text { (syst) }
$$


and

$$
A_{C P}\left(B_{s} \rightarrow K \pi\right)=0.27 \pm 0.08 \text { (stat) } \pm 0.02 \text { (syst). }
$$

The result for $A_{C P}\left(B_{d} \rightarrow K \pi\right)$ constitutes the most precise measurement available to date. The significance of the measured deviation from zero exceeds $6 \sigma$, making this the first observation (greater than $5 \sigma$ ) of $C P$ violation in the $B$ meson sector at a hadron collider. The same significance of $A_{C P}\left(B_{s} \rightarrow K \pi\right)$ is $3.3 \sigma$, therefore this is the first evidence for $C P$ violation in the decays of $B_{s}^{0}$ mesons.

\section{Time-dependent study of $B_{d} \rightarrow \pi \pi$ and $B_{s} \rightarrow K K$}

The time-repent study os charmless charged two-body $B$ decays allows to probe the picture of $C P$ violation in the SM [5, 6, 7].

Here we present the time-dependent $C P$ asymmetries at LHCb using decays to $C P$ eigenstates, namely $B_{d} \rightarrow \pi^{+} \pi^{-}$and $B_{s}^{0} \rightarrow K^{+} K^{-}$[8]. The former analysis was already performed by BABAR [9] and Belle [10], whereas the latter is a novelty.

The time-dependent $C P$ asymmetry for a $C P$ eigenstate $f$ can be written as

$$
A_{C P}(t)=\frac{A_{f}^{\mathrm{dir}} \cos (\Delta m t)+A_{f}^{\mathrm{dir}} \cos (\Delta m t)}{\cosh \left(\frac{\Delta \Gamma}{2} t\right)-A_{f}^{\Delta \Gamma} \sinh \left(\frac{\Delta \Gamma}{2} t\right)}
$$

where $A_{f}^{\mathrm{dir}}$ and $A_{f}^{\mathrm{mix}}$ parametrize direct and mixing-induced CP violation respectively, and the quantity $A_{f}^{\Delta \Gamma}$ is constrained by the consistency relation

$$
\left(A_{f}^{\mathrm{dir}}\right)^{2}+\left(A_{f}^{\mathrm{mix}}\right)^{2}+\left(A_{f}^{\Delta \Gamma}\right)^{2}=1
$$

For a detailed description of these variables see for example Chap. 3 of the LHCb roadmap document [11].

We select the events using the criteria described in previous section for the $B_{d} \rightarrow K \pi$ and than apply PID selection in order to obtain three independent sets passing through $K \pi, \pi \pi$ and $K K$ hypotheses. The $K \pi$ events are used to calibrate the opposite side tagging performance. For the calibration we split the the sample into 5 categories each consisting of approximately equal amount of events. We then perform a simultaneous maximum likelihood (ML) fit to the decay time and invariant mass to extract the mistag probabilities with their likelihoods for each category. These probabilities are then inserted into the $B_{d} \rightarrow \pi \pi$ and $B_{s} \rightarrow K K$ ML fits. The fit models for each hypothesis include the signal, cross-feed background from two-body charmless decays, combinatorial background and partially reconstructed 3-body background. The projections of the fit can be seen in Fig. 2.

The results for the $B_{d} \rightarrow \pi \pi$ are

$$
\begin{aligned}
& A^{\mathrm{dir}}=0.11 \pm 0.21(\text { stat }) \pm 0.03(\text { syst }), \\
& A^{\text {mix }}=0.56 \pm 0.17(\text { stat }) \pm 0.03 \text { (syst) }
\end{aligned}
$$

where the statistical correlation between the two is found to be $34 \%$. They are in agreement with world average from the B factories. The result for $B_{s} \rightarrow K K$

$$
\begin{aligned}
& A^{\mathrm{dir}}=0.02 \pm 0.18(\text { stat }) \pm 0.04(\text { syst }), \\
& A^{\text {mix }}=0.17 \pm 0.18(\text { stat }) \pm 0.05(\text { syst }),
\end{aligned}
$$



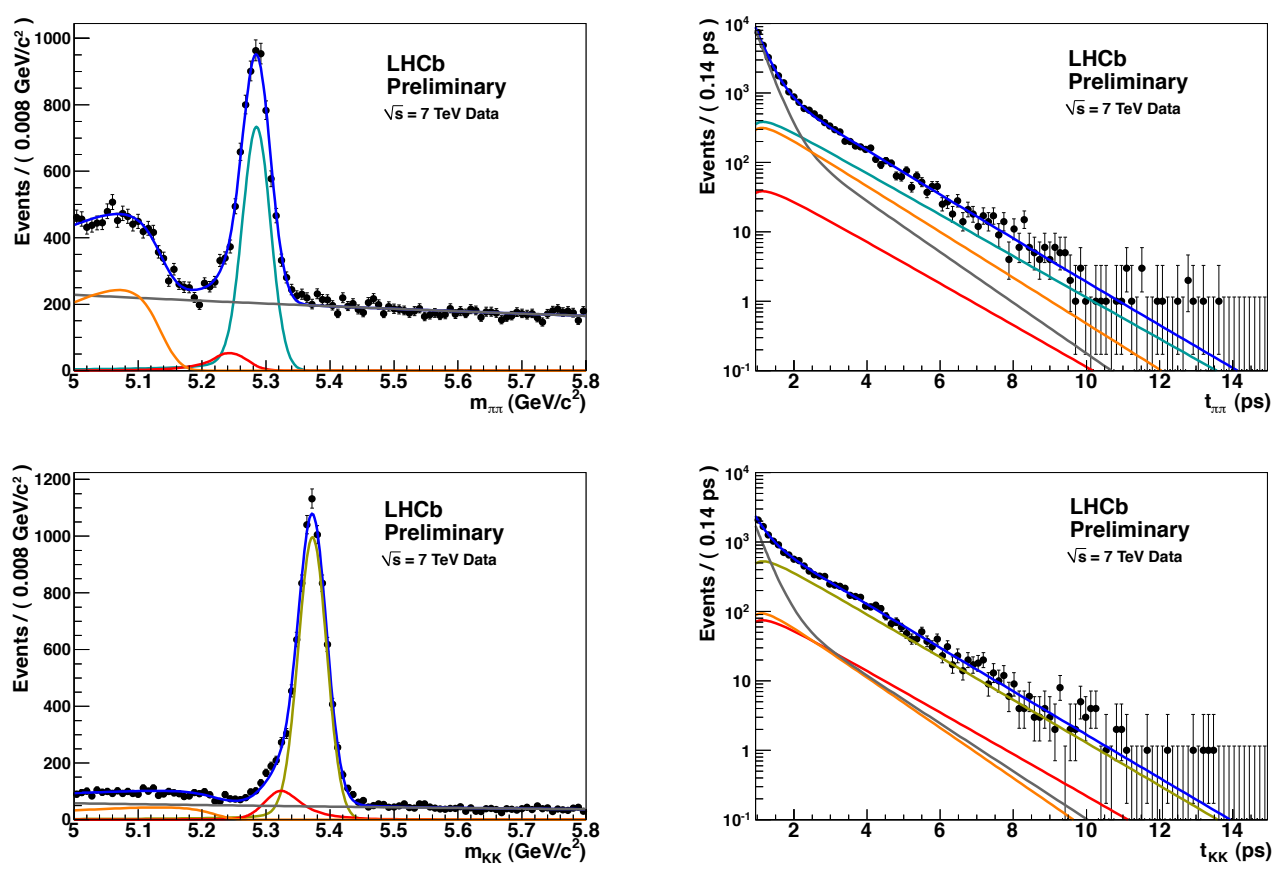

Figure 2: : Invariant mass (left) and decay time (right) projections for the $B_{d} \rightarrow \pi \pi$ (top) and $B_{s} \rightarrow K K$ (bottom) decays. The data are described by the overall fit result (solid blue line) which is the sum of: $B_{d} \rightarrow \pi \pi$ signal (dotted light blue line), $B_{s} \rightarrow K K$ signal (dotted dark yellow line); $B_{d} \rightarrow K \pi$ cross-feed background (dashed double-dotted red line); partially reconstructed three-body (dashed orange line) and combinatorial (dashed dotted grey line) backgrounds.

with a statistical correlation of $10 \%$.

\section{Effective $B_{S} \rightarrow K K$ lifetime studies}

When the initial flavor of the $B_{s}$ meson is unknown the decay time distribution can be written as

$$
\Gamma(t) \propto\left(1-\mathscr{A}_{\Delta \Gamma_{s}}\right) e^{-\Gamma_{L} t}+\left(1+\mathscr{A}_{\Delta \Gamma_{s}}\right) e^{-\Gamma_{H} t} .
$$

The quantities $\Gamma_{\mathrm{H}}$ and $\Gamma_{\mathrm{L}}$ are the decay widths of the heavy and light $B_{s}$ mass eigenstates and $\Delta \Gamma_{s}=$ $\Gamma_{\mathrm{L}}-\Gamma_{\mathrm{H}}$ is the decay width difference. The parameter $\mathscr{A}_{\Delta \Gamma_{s}}$ is defined as $\mathscr{A}_{\Delta \Gamma_{s}}=-2 \operatorname{Re}(\lambda) /\left(1+|\lambda|^{2}\right)$ where $\lambda=(q / p)(\bar{A} / A)$, where the complex coefficients $p$ and $q$ define the mass eigenstates of the $B_{S}-\bar{B}_{S}$ system in terms of the flavor eigenstates (see e.g., Ref. [12]) and $A(\bar{A})$ is the amplitude for a $B_{s}\left(\bar{B}_{s}\right)$ meson to decay to the $K^{+} K^{-}$final state.

If the decay time distribution given by Eq. 4.1 is fitted with a single exponential function the effective lifetime is given by [13]

$$
\tau_{K K}=\frac{\tau_{B_{s}}}{1-y_{s}^{2}}\left[\frac{1+2 \mathscr{A}_{\Delta \Gamma_{s}} y_{s}+y_{s}^{2}}{1+\mathscr{A}_{\Delta \Gamma_{s}} y_{s}}\right]=\tau_{B_{s}}\left(1+\mathscr{A}_{\Delta \Gamma_{s}} y_{s}+\mathscr{O}\left(y_{s}^{2}\right)\right),
$$

where $\tau_{B_{s}}=2 /\left(\Gamma_{H}+\Gamma_{L}\right)=\Gamma_{s}^{-1}$ and $y_{s}=\Delta \Gamma_{s} / 2 \Gamma_{s}$. The $K^{+} K^{-}$final state is $C P$-even and so in the SM the decay is dominated by the light mass eigenstate such that $\mathscr{A}_{\Delta \Gamma_{s}}=-0.972 \pm 0.012$ [7, 14] 
and the effective lifetime thus is approximately equal to $\Gamma_{\mathrm{L}}^{-1}$. Adopting the approach of Ref. [6] and using the world averages of $\Gamma_{s}$ and $\Delta \Gamma_{s}[15]$ and the SM prediction of $\mathscr{A}_{\Delta \Gamma_{s}}$, the effective lifetime is predicted to be $\tau_{K K}=1.40 \pm 0.02 \mathrm{ps}$.

Details of the analysis performed by LHCb can be found in Ref. [16]. We extract the effective $B_{s} \rightarrow K K$ lifetime using an unbinned log-likelihood fit. A fit to the invariant mass spectrum is performed to determine the sWeights [22] that are used to isolate the $B_{s} \rightarrow K K$ decay time distribution from the residual background. The contribution from the $B_{d} \rightarrow K \pi$ decay is separated by demanding tight requirements on the particle identification properties of the final state particles.

The fit finds $997 \pm 34 B_{s} \rightarrow K K$ decays. Figure 4(a) shows the resulting invariant mass spectrum for $B_{s} \rightarrow K K$ candidates. Figure 4(b) shows the fit corresponding to the decay time distribution of $B_{s} \rightarrow K K$ signal. The effective decay time is found to be:

$$
\tau_{K K}=1.455 \pm 0.046 \text { (stat.) } \pm 0.006 \text { (syst.) ps, }
$$

in good agreement with the SM prediction of $1.40 \pm 0.02$. The leading source of systematics is the reconstruction efficiency.
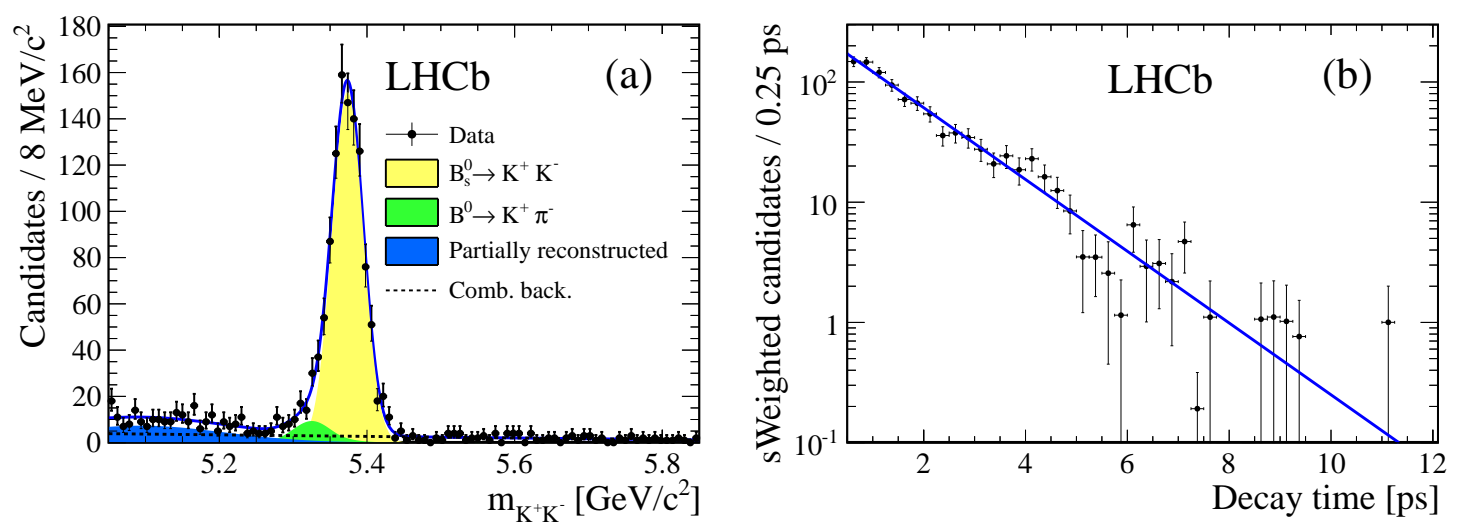

Figure 3: (a) Invariant mass spectrum for all selected $B_{s} \rightarrow K K$ candidates. (b) Decay time distribution of $B_{s} \rightarrow K K$ signal extracted using sWeights and the fitted exponential function.

Using the value of the $B^{0}$ mass [12] as input, the $B^{0}$ lifetime is found to be

$$
\tau_{B d}=1.536 \pm 0.031 \text { (stat.) ps }
$$

which agrees with the current world-average $\tau_{B_{d}}=1.519 \pm 0.007 \mathrm{ps}$ [12].

\section{5. $B_{S} \rightarrow \phi \phi$ triple asymmetries}

Another way to test the SM are the studies of the polarization amplitudes and triple product asymmetries in the $B_{S} \rightarrow \phi \phi$ decay $[17,18,19]$. There are three possible spin configurations of the vector mesons allowed by conservation of angular momentum. These can be written as linear polarization states $A_{0}, A_{\|}$and $A_{\perp}$. The final state is a superposition of $C P$-even and $C P$-odd states. The longitudinal $\left(A_{0}\right)$ and parallel $\left(A_{\|}\right)$components are $C P$-even while the perpendicular $\left(A_{\perp}\right)$ component is $C P$-odd. To measure polarization amplitudes, a time-integrated untagged angular analysis is 
performed [20]. Due to fast oscillation of the $B_{s}$ meson an equal number of $B_{s}$ and $\bar{B}_{s}$ are assumed at production. In addition, the $C P$ violating phase is assumed to be zero, as predicted in Ref. [21]. Under these assumptions, the differential decay width is given by:

$$
\begin{array}{r}
\frac{32 \pi}{9} \frac{d^{3} \Gamma}{d \cos \theta_{1} d \cos \theta_{2} d \Phi}=\frac{4}{\Gamma_{L}}\left|A_{0}\right|^{2} \cos ^{2} \theta_{1} \cos ^{2} \theta_{2}+\frac{1}{\Gamma_{L}}\left|A_{\|}\right|^{2} \sin ^{2} \theta_{1} \sin ^{2} \theta_{2}(1+\cos 2 \Phi) \\
+\frac{1}{\Gamma_{H}}\left|A_{\perp}\right|^{2} \sin ^{2} \theta_{1} \sin ^{2} \theta_{2}(1-\cos 2 \Phi)+\frac{\sqrt{2}}{\Gamma_{L}} \operatorname{Im}\left(A_{0} A_{\|}^{*}\right) \sin 2 \theta_{1} \sin 2 \theta_{2} \cos \Phi
\end{array}
$$

where the helicity angles $\left(\theta_{1}, \theta_{2}, \Phi\right)$ are defined in Fig. 5 and $\Gamma_{L, H}$ are the lifetimes for the light and heavy mass eigenstates respectively. The strong phase difference $\delta_{\|}$is defined as $\arg \left(A_{\|} / A_{0}\right)$.

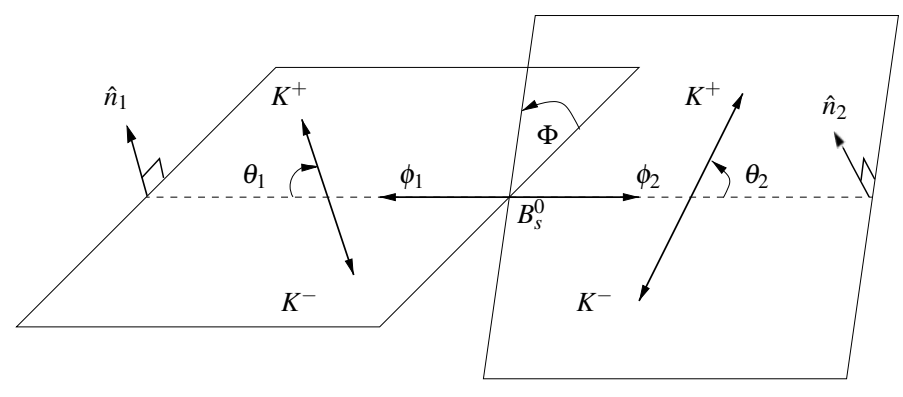

Figure 4: Helicity angles for the decay $B_{s} \rightarrow \phi \phi$ where $\theta_{1,2}$ is the angle between the $K^{+}$track momentum in the $\phi_{1,2}$ meson rest frame and the parent $\phi_{1,2}$ momentum in the $B_{s}$ rest frame, $\Phi$ is the angle between the two $\phi$ meson decay planes and $\hat{n}_{1,2}$ is the unit vector normal to the decay plane of the $\phi_{1,2}$ meson.

Non zero triple product asymmetries can be due to either T violation or final state interactions. The former, assuming $C P T$ conservation, implies $C P$ is violated. In the decay $B_{s} \rightarrow \phi \phi$ two triple products can be constructed, denoted $U=\sin (2 \Phi) / 2$ and $V= \pm \sin (\Phi)$, where the positive sign is taken if the T-even quantity $\cos \theta_{1} \cos \theta_{2} \geq 0$ and the negative sign otherwise. These correspond to the T-odd triple products

$$
\sin \Phi=\left(\hat{n_{1}} \times \hat{n_{2}}\right) \cdot \hat{p_{1}}, \sin (2 \Phi) / 2=\left(\hat{n_{1}} \cdot \hat{n_{2}}\right)\left(\hat{n_{1}} \times \hat{n_{2}}\right) \cdot \hat{p_{1}}
$$

where $\hat{n}_{i}(\mathrm{i}=1,2)$ is a unit vector perpendicular to the $\phi$ decay plane and $\hat{p}_{1}$ is a unit vector in the direction of the $\phi$ momentum in the $B_{s}$ rest frame (see Fig. 5).

The asymmetries are defined as

$$
A_{U}=\frac{N_{+}-N_{-}}{N_{+}+N_{-}}, A_{V}=\frac{M_{+}-M_{-}}{M_{+}+M_{-}}
$$

where $N_{+}\left(N_{-}\right)$is the number of events with $U>0(U<0)$ and $M_{+}\left(M_{-}\right)$is the number of events with $V>0(V<0)$.

$B_{s} \rightarrow \phi \phi$ candidates are selected using events where both $\phi$ mesons decay into the final state $K^{+} K^{-}$. The candidate selection criteria are optimized using a data-driven approach based on the ${ }_{s} \mathscr{P}$ lot technique [22] with the invariant mass of the four-kaon system as the discriminating variable to separate signal from background. 
To determine the triple product asymmetries, the dataset is partitioned according to whether $U$ $(V)$ is less than or greater than zero. Simultaneous fits are performed to the mass distributions for each of the two partitions. In these fits, the mean and resolution of the Gaussian signal component together with the slope of the exponential background component are common parameters. The asymmetries are left as free parameters and are fitted for directly in the simultaneous fit.

The measurements of the polarization amplitudes and triple product asymmetries are:

$$
\begin{aligned}
\left|A_{0}\right|^{2} & =0.365 \pm 0.022 \text { (stat) } \pm 0.012 \text { (syst) } \\
\left|A_{\perp}\right|^{2} & =0.291 \pm 0.024 \text { (stat) } \pm 0.010 \text { (syst) } \\
\left|A_{\|}\right|^{2} & =0.344 \pm 0.024(\text { stat }) \pm 0.014 \text { (syst) } \\
\cos \left(\delta_{\|}\right) & =-0.844 \pm 0.068 \text { (stat) } \pm 0.029 \text { (syst) } \\
A_{U} & =-0.055 \pm 0.036(\text { stat }) \pm 0.018 \text { (syst) } \\
A_{V} & =0.010 \pm 0.036 \text { (stat) } \pm 0.018 \text { (syst) }
\end{aligned}
$$

The leading systematic sources are the acceptances and the S-wave contributions. The measured values agree well with previous measurements by the CDF collaboration [23]. The triple product asymmetries are consistent with zero and hence no indication of T-odd asymmetries are observed with the present statistics.

\section{References}

[1] LHCb collaboration, A. A. Alves Jr. et al., JINST 3 (2008) S08005.

[2] R. Aaij et al. [LHCb Collaboration], Phys. Rev. Lett. 108 (2012) 201601 [arXiv:1202.6251 [hep-ex]].

[3] E. Baracchini and G. Isidori, Phys. Lett. B 633 (2006) 309 [hep-ph/0508071].

[4] H. Albrecht et al. [ARGUS Collaboration], Phys. Lett. B 229, 304 (1989).

[5] R. Fleischer, Phys. Lett. B 459 (1999) 306 [arXiv:hep-ph/9903456].

[6] R. Fleischer, Eur. Phys. J. C 52 (2007) 267 [arXiv:0705.1121 [hep-ph]].

[7] R. Fleischer and R. Knegjens, arXiv:1011.1096 [hep-ph].

[8] LHCb collaboration, CERN-LHCb-CONF-2012-007.

[9] B. Aubert et al. [BaBar Collaboration], arXiv:0807.4226 [hep-ex].

[10] H. Ishino et al. [Belle Collaboration], Phys. Rev. Lett. 98 (2007) 211801 [arXiv:hep-ex/0608035].

[11] B. Adeva et al. [The LHCb Collaboration], arXiv:0912.4179 [hep-ex].

[12] Particle Data Group, J. Beringer et al., Phys. Rev. D86 (2012) 010001.

[13] K. Hartkorn and H. Moser, Eur. Phys. J. C8 (1999) 381.

[14] K. de Bruyn et al., Phys. Rev. D86 (2012) 014027.

[15] Heavy Flavor Averaging Group, D. Asner et al., [arXiv:1010.1589].

[16] RAaij et al. [LHCb Collaboration], Phys. Lett. B 716, 393 (2012) [arXiv:1207.5993 [hep-ex]].

[17] M. Gronau and J. L. Rosner, Phys. Rev. D 84, 096013 (2011). 
[18] W. Bensalem and D. London, Phys. Rev. D 64, 116003 (2001).

[19] A. Datta and D. London, Int. J. Mod. Phys. A19, 2505 (2004).

[20] R. Aaij et al. [LHCb Collaboration], Phys. Lett. B 713, 369 (2012) [arXiv:1204.2813 [hep-ex]].

[21] M. Raidal, Phys. Rev. Lett. 89, 231803 (2002).

[22] M. Pivk and F. R. L. Diberder, Nucl. Instr. Methods A555, 356 (2005).

[23] D. Acosta et al. [CDF Collaboration], Phys. Rev. Lett. 95 (2005) 031801 [hep-ex/0502044]. 